Case Study

\title{
Use of an arm weight-bearing combined with upper-limb reaching apparatus to facilitate motor paralysis recovery in an incomplete spinal cord injury patient: a single case report
}

\author{
Takashi Hoei, OT, MS (1)* $^{*}$ Kazumi Kawahira, MD, PhD2), Hidefumi Fukuda, PT, MS ${ }^{1)}$, \\ Keizo Sihgenobu, MD ${ }^{1)}$, Megumi Shimodozono, MD, PhD²), Tadashi Ogura, MD ${ }^{1)}$ \\ 1) Kohshinkai Ogura Hospital: 27-22 Kasanohara-cho, Kanoya-shi, Kagoshima 893-0023, Japan \\ 2) Department of Rehabilitation and Physical Medicine, Graduate School of Medical and Dental \\ Sciences, Kagoshima University, Japan
}

\begin{abstract}
Purpose] Training using an arm weight-bearing device combined with upper-limb reaching apparatus to facilitate motor paralysis recovery, named the "Reaching Robot", as well as Repetitive Facilitation Exercise were applied to a patient with severe impairment of the shoulder and elbow due to incomplete spinal cord injury and the effects were examined. [Subjects and Methods] A 66-year-old man with incomplete spinal cord injury participated in an upper extremity rehabilitation program involving a Reaching Robot. The program was comprised of active motor suspension, continuous low amplitude neuromuscular electrical stimulation and functional vibratory stimulation, as well as Repetitive Facilitation Exercise combined with continuous low amplitude neuromuscular electrical stimulation. This protocol used a crossover design following an $A_{1}-B_{1}-A_{2}-B_{2}$. "A" consisted of 2 weeks of Repetitive Facilitation Exercise, and "B" consisted of 2 weeks of Reaching Robot training. [Results] Improvements were observed after all sessions. Active range of motion for shoulder flexion improved after 2 weeks of Reaching Robot sessions only. There were no adverse events. [Conclusion] Reaching Robot training for severe paretic upperextremity after incomplete spinal cord injury was a safe and effective treatment. Reaching Robot training may be useful for rehabilitation of paretic upper-extremity after incomplete spinal cord injury.

Key words: Incomplete spinal cord injury, Reaching Robot training, Repetitive Facilitation Exercise
\end{abstract}

(This article was submitted Oct. 14, 2016, and was accepted Nov. 11, 2016)

\section{INTRODUCTION}

As individuals with tetraplegia caused by incomplete spinal cord injury (SCI) are often unable to use their arm and hands, quality of life decreases ${ }^{1}$. Although various upper extremity rehabilitation methods have been applied to date, the functional effects have been limited.

Recent evidence has shown that intensive repetition of movement could make a useful contribution to clinical practice ${ }^{2)}$, constituting a promising approach for patients affected by $\mathrm{SCI}^{3)}$. However, independent movement practice is particularly difficult for individuals who are unable to lift the arm against gravity or have minimal hand movement ability.

It is expected that Robot-assisted training will contribute to promoting motor recovery due to its ability to deliver highly reproducible controlled movement sequences ${ }^{4,5)}$, but these benefits in the upper extremity currently remain insufficient ${ }^{6}$. Although a Robot with assisted-as-needed control (Robot) can aid paretic limb movement ${ }^{7}$, , such Robots cannot directly facilitate realization of the intended limb movement. Neuromuscular electrical stimulation and vibratory stimulation have

*Corresponding author. Takashi Hoei (E-mail: Htakashi.kagoshima@gmail.com)

(C2017 The Society of Physical Therapy Science. Published by IPEC Inc.

This is an open-access article distributed under the terms of the Creative Commons Attribution Non-Commercial No Derivatives (by-nc-nd) License $<$ http://creativecommons.org/licenses/by-nc-nd/4.0/>. 
shown facilitation effects when these stimulations are applied to effective points on paretic limbs and synchronized with initiation of the patient's intentional movement ${ }^{8-10)}$. Continuous low amplitude neuromuscular electrical stimulation (CNMES) maintains the lower motor tracts in a state that reacts to motor commands from upper motor tracts, similar to synchronized initiation of intended movements by the patient. CNMES during training might be useful if it is applied continuously to the targeted musculature rather than in an on/off mode using either electromyography- or position-triggering ${ }^{8)}$.

Functional vibratory stimulation $(\mathrm{FVS})^{10}$, which provides vibratory stimulation synchronized with initiation of the patient's intentional movement, could also be an encouraging add-on approach to Robot for paretic upper limb.

This simultaneous application of CNMES and FVS during Robot training has not been reported previously, and it is considered that a skilled technique is necessary to carry it out.

An active arm weight-bearing system that has intellectual sensing and driving functions that control motion and force and can be used in training to improve movement of the hemiplegic arm ${ }^{11)}$. The system reduces the gravity on the arm by partially supporting the weight of the subject's arm during hemiplegic arm rehabilitation training, can enable the subject to handle objects more easily. The study used an arm weight-bearing device combined with an upper-limb reaching apparatus to facilitate motor paralysis recovery, named the "Reaching Robot" (Yasukawa Electric Co., Ltd. Fukuoka) which was developed by the Department of Rehabilitation and Physical Medicine, Graduate School of Medical and Dental Sciences and the Department of Mechanical Engineering, Graduate School of Science and Engineering, Kagoshima University and Yasukawa Electric Corporation.

Training using the Reaching Robot with CNMES and FVS to facilitate realization of intended movement is expected to provide more effective therapy for severe impairment of the shoulder and elbow in a patient with incomplete SCI or brain injury. In this case report, improvements of shoulder and elbow function after Reaching Robot training were compared to those after Repetitive Facilitation Exercise $(\mathrm{RFE})^{12)}$ combined with $\mathrm{CNMES}^{8)}$ in a patient with incomplete SCI.

\section{SUBJECTS AND METHODS}

The patient was a 66-year-old right-handed man with tetraplegia subsequent to a fall. The patient had undergone C3-C4 fusion and C5-C6 laminoplasty and was admitted to this hospital 59 days after the onset of SCI. On admission, the patient showed minimal voluntary movements of the upper limbs (American Spinal Injury Association Motor Score: left arm 12/25, right arm 20/25). Immediately after admission to this hospital, the patient began receiving daily rehabilitation with RFE; this study was started 4 weeks after admission.

The rehabilitation protocol followed an $\mathrm{A}_{1}-\mathrm{B}_{1}-\mathrm{A}_{2}-\mathrm{B}_{2}$ schedule. The "A"-period consisted of 2 weeks of RFE combined with CNMES to the proximal and distal upper extremity for 20-minutes each. The "B"-period consisted of 2 weeks of Reaching Robot training and RFE combined with CNMES to the distal upper extremity for 20-minutes each. All sessions lasted for 40 minutes and were performed 7-days/week. This study was approved by the ethics committees of Kohshinkai Ogura Hospital (No 2015-A3) and written informed consent was obtained from the patient.

The Reaching Robot constructed in a motor-load-relieving apparatus combined with upper-limb reaching apparatus to facilitate motor paralysis recovery (Fig. 2). Two aspects of the Reaching Robot can be adjusted to alter the degree of difficulty: (1) the amount of motor-load-relief; and (2) the height and distance of the target-button and start-button. In addition to these functions to enable repetitive reaching-task, the Reaching Robot can facilitate the patient's ability to reach while FVS and CNMES are applied to the anterior part of the deltoid and superior part of the trapezius. The FVS device is attached vertically to the target muscle belly and FVS $(100 \mathrm{~Hz})$ begins when the start-button is pushed, then halted when the targetbutton is pushed. CNMES (symmetrical biphasic waveform, pulse width $250 \mu \mathrm{s}, 50 \mathrm{~Hz}$ ) was delivered using a portable surface neuromuscular stimulator (ITO ESPURGE, Ito Co., Ltd. Tokyo). The intensity of the electrical current was adjusted to produce a slight contraction of the target muscle without inducing obvious limb/joint movement at rest before training.

Reaching Robot training was performed under the supervision of an occupational therapist, who determined the difficulty adjustments appropriate for the patient. At the start of training, the patient was instructed on smooth reaching. After each session, the patient received feedback on the number of repetitions.

The RFE program, a combination of high repetition rate and neurofacilitation, is a recently developed approach to paresis or dysfunctions due to neurological diseases ${ }^{2,13,14)}$ and a randomized controlled trial (RCT) study ${ }^{12}$ ) showed its effectiveness for patients with hemiplegia following stroke. This technique involved the use of rapid passive stretching of the muscles in conjunction with tapping and rubbing the skin to facilitate realization of the patient's intended movement. Patients were directed to concentrate on generating movement of the targeted joint ${ }^{15,16)}$.

As with the Reaching Robot training protocol, RCT study ${ }^{8)}$ has shown that RFE with CNMES is effective for patients with severe hemiplegia following stroke.

Outcome measures were the knee-mouth test and the finger test of the Stroke Impairment Assessment Set (SIAS) ${ }^{17)}$ for evaluating paralysis (rating: 0 to 5), the Simple Test for Evaluating Hand Function (STEF) ${ }^{13,18)}$ for the ability to manipulate objects (rating: 0 to 100), Active range of motion (Active ROMs) for flexion at the shoulder and elbow and for extension at the left wrist, and the Modified Ashworth Scale (MAS) ${ }^{19)}$ for spasticity in the elbow flexor and wrist flexor (rating: 0 to 5). Using recorded evaluations obtained at the beginning and the end of each session, these test results were compared by the occupational therapist supervising treatment as well as another therapist not directly involved in the treatment in order to guarantee objectivity. 


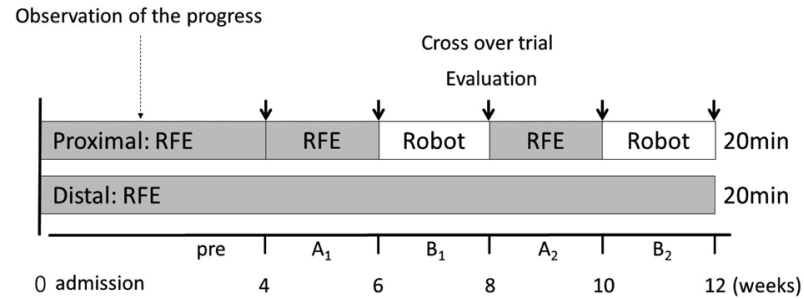

Fig. 1. Experimental procedure during the 12-week study period. Before and after each of the two-week sessions $\left(\mathrm{A}_{1}, \mathrm{~B}_{1}, \mathrm{~A}_{2}\right.$, $\mathrm{B}_{2}$ ), motor function, spasticity and the ability to manipulate objects were evaluated by SIAS, Active ROMs, MAS and STEF.

RFE: Repetitive Facilitation Exercise; Robot: Reaching Robot training; pre: pretest; SIAS: Stroke Impairment Assessment Set; Active ROMs: Active range of motion; MAS: Modified Ashworth Scale; STEF: Simple Test for Evaluating Hand Function
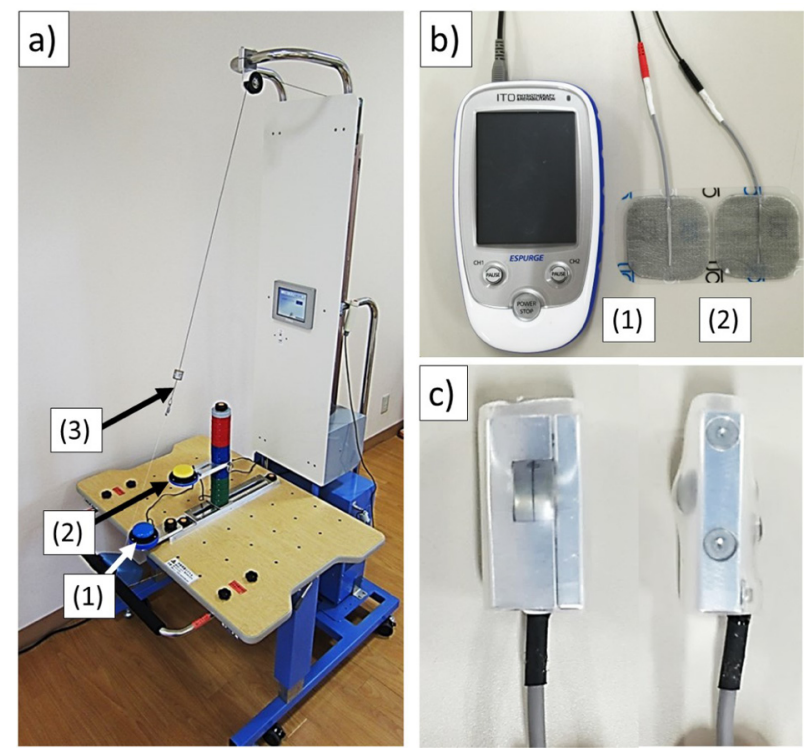

Fig. 2. Components of the Reaching Robot training system a. Reaching: (1) start-button, (2) target-button, (3) suspension wire; b. CNMES device: (1) ITO ESPURGE, (2) electrode pads $(50 \mathrm{~mm}$ $\times 50 \mathrm{~mm}$ );

c. FVS device: $33 \mathrm{~mm}$ long $\times 15 \mathrm{~mm}$ wide $\times 7 \mathrm{~mm}$ high).

\section{RESULTS}

The patient was able to perform more repetitions of exercises for the shoulder joint and elbow joint during Reaching Robot training (approximately 400-450) than during RFE combined with CNMES to the proximal upper extremity (approximately 200-250).

The protocol was well tolerated by the patient. There were no observable adverse effects related to either Reaching Robot training or RFE combined with CNMES. Upper extremity function improved on all outcome measures except for MAS of the wrist flexor. The patient improved by one point on the SIAS knee-mouth test and finger test after session $A_{1}$ and one point on the knee-mouth test only after session $B_{1}$. There were no subsequent changes on SIAS. Active ROMs improved by $10^{\circ}$ for both elbow flexion and wrist extension after session $A_{1}$. After session $B_{1}$, Active ROMs for both shoulder and elbow flexion improved further, particularly $20^{\circ}$ for elbow flexion. After session $\mathrm{B}_{2}$, the increase in shoulder flexion was $10^{\circ}$. STEF improved one point after session $\mathrm{B}_{2}$ only. MAS at elbow flexor improved one point after session $\mathrm{A}_{2}$ only (Fig. 3, 4).

\section{DISCUSSION}

The patient was diagnosed with SCI on Magnetic Resonance Imaging. In addition, it was approximately three months after the onset and the patient could not expect spontaneous recovery ${ }^{20,21)}$.

This single case study demonstrates preliminary results of Reaching Robot training and RFE combined with CNMES for rehabilitation of upper extremity movements following incomplete SCI. The results showed that both Reaching Robot training and RFE combined with CNMES could achieve similar magnitudes of motor improvement on knee-mouth test of SIAS and Active ROMs of elbow flexion. However, Active ROMs of shoulder flexion were improved following Reaching Robot training only (session $B_{1}, B_{2}$ ). These findings suggest that Reaching Robot training can be safely implemented for treatment of upper extremity motor function in a subject with incomplete SCI.

Although both training programs facilitated a high number of repetitions of active movement of the proximal upper extremity, there were 400-450 repetitions during a 20-minute Reaching Robot training session compared to 200-250 repetitions during RFE combined with CNMES. This difference in the number of repetitions might have produced the difference in improvement in Active ROMs of shoulder flexion.

A recent systematic review showed that exercise therapy and functional electrical stimulation improved arm and hand function in patients with $\mathrm{SCI}^{6}$. When CNMES, which can elicit network response is combined with FVS, which enhances somatosensory processing in the lower motor system ${ }^{10)}$, these two stimulations could potentiate a facilitating effect and become useful tools for neuromodulation. Thus, simultaneous combination of CNMES and FVS in this case study is a novel treatment and is expected to contribute to future developments in rehabilitation. 


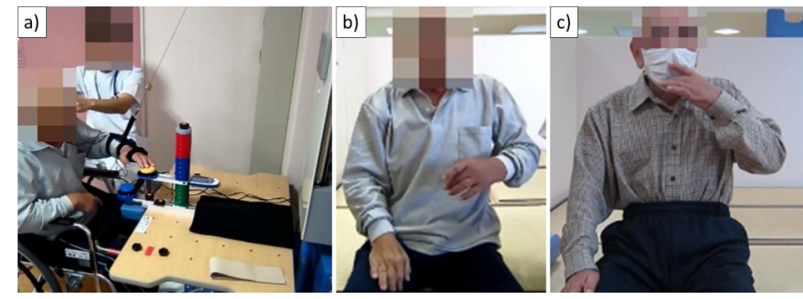

Fig 3. Reaching Robot training and motor improvement on SIAS Knee-mouth test

a) The patient during Reaching Robot training. b) SIAS Kneemouth test before treatment. c) SIAS Knee-mouth test after 12 weeks of upper extremity training using Reaching Robot and RFE. SIAS: Stroke Impairment Assessment Set; RFE: Repetitive Facilitation Exercise
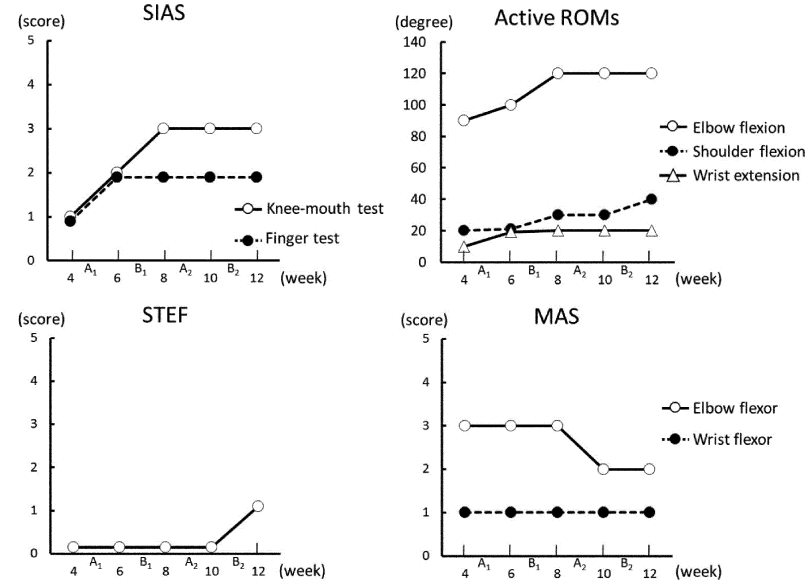

Fig 4. Time course of changes in evaluation scores after sessions $\mathrm{A}_{1}, \mathrm{~B}_{1}, \mathrm{~A}_{2}, \mathrm{~B}_{2}$. Figure 1 shows improvement in upper extremity function on SIAS, Active ROMs, STEF and MAS Upper extremity function improved on all outcome measures except MAS of the wrist flexor. Active ROMs for shoulder flexion improved only after B1 and B2.

SIAS: Stroke Impairment Assessment Set; Active ROMs: Active range of motion; MAS, Modified Ashworth Scale; STEF: Simple Test for Evaluating Hand Function

According to the so-called Hebbian theory ${ }^{22)}$, in order to promoting functional recovery of paretic limbs or motor learning, in other words, strengthening a target neural circuit relating to intended movement of the patient, it is necessary to apply excitation repeatedly in order to realize the patient's targeted intentional movement. As methods to facilitate realization of these targeted intentional movements, RFE using CNMES and Reaching Robot using both CNMES and FVS may provide many advantages.

\section{Conflict of interest}

The author(s) declared the following potential conflicts of interest with respect to the research, authorship, and/or publication of this article: T. Hoei receives a study fund and the loan of the apparatus from Yasukawa Electric CO (Fukuoka, Japan). K. Kawahira is an adviser of Yasukawa Electric Co (Fukuoka, Japan). M. Shimodozono receives a study fund from Yasukawa Electric Co (Fukuoka, Japan).

\section{REFERENCES}

1) Snoek GJ, IJzerman MJ, Hermens HJ, et al.: Survey of the needs of patients with spinal cord injury: impact and priority for improvement in hand function in tetraplegics. Spinal Cord, 2004, 42: 526-532. [Medline] [CrossRef]

2) Kawahira K, Shimodozono M, Etoh S, et al.: Effects of intensive repetition of a new facilitation technique on motor functional recovery of the hemiplegic upper limb and hand. Brain Inj, 2010, 24: 1202-1213. [Medline] [CrossRef]

3) Zariffa J, Kapadia N, Kramer JL, et al.: Feasibility and efficacy of upper limb robotic rehabilitation in a subacute cervical spinal cord injury population. Spinal Cord, 2012, 50: 220-226. [Medline] [CrossRef]

4) Norouzi-Gheidari N, Archambault PS, Fung J: Effects of robot-assisted therapy on stroke rehabilitation in upper limbs: systematic review and meta-analysis of the literature. J Rehabil Res Dev, 2012, 49: 479-496. [Medline] [CrossRef]

5) Kwakkel G, Kollen BJ, Krebs HI: Effects of robot-assisted therapy on upper limb recovery after stroke: a systematic review. Neurorehabil Neural Repair, 2008, 22: 111-121. [Medline] [CrossRef]

6) Lu X, Battistuzzo CR, Zoghi M, et al.: Effects of training on upper limb function after cervical spinal cord injury: a systematic review. Clin Rehabil, 2015, 29: 3-13. [Medline] [CrossRef]

7) $\mathrm{Hu} \mathrm{XL}$, Tong RK, Ho NS, et al.: Wrist rehabilitation assisted by an electromyography-driven neuromuscular electrical stimulation robot after stroke. Neurorehabil Neural Repair, 2015, 29: 767-776. [Medline] [CrossRef]

8) Shimodozono M, Noma T, Matsumoto S, et al.: Repetitive facilitative exercise under continuous electrical stimulation for severe arm impairment after subacute stroke: a randomized controlled pilot study. Brain Inj, 2014, 28: 203-210. [Medline] [CrossRef]

9) Fujiwara T, Kasashima Y, Honaga K, et al.: Motor improvement and corticospinal modulation induced by hybrid assistive neuromuscular dynamic stimulation (HANDS) therapy in patients with chronic stroke. Neurorehabil Neural Repair, 2009, 23: 125-132. [Medline] [CrossRef] 
10) Shirahashi I, Matsumoto S, Shimodozono M, et al.: Functional vibratory stimulation on the hand facilitates voluntary movements of a hemiplegic upper limb in a patient with stroke. Int J Rehabil Res, 2007, 30: 227-230. [Medline] [CrossRef]

11) Yu Y, Nagai M, Matuda J, et al.: Control Strategy of an Active Arm Weight-Bearing Unit for Hemiplegic Arm Rehabilitation. IEEE International Conference on Information and Automation, 2014: 812-818.

12) Shimodozono $M$, Noma $T$, Nomoto $Y$, et al.: Benefits of a repetitive facilitative exercise program for the upper paretic extremity after subacute stroke: a randomized controlled trial. Neurorehabil Neural Repair, 2013, 27: 296-305. [Medline] [CrossRef]

13) Kawahira K, Noma $T$, Iiyama J, et al.: Improvements in limb kinetic apraxia by repetition of a newly designed facilitation exercise in a patient with corticobasal degeneration. Int J Rehabil Res, 2009, 32: 178-183. [Medline] [CrossRef]

14) Usuki F, Tohyama S: Three case reports of successful vibration therapy of the plantar fascia for spasticity due to cerebral palsy-like syndrome, fetal-type minamata disease. Medicine (Baltimore), 2016, 95: e3385. [Medline] [CrossRef]

15) Kawahira K, Shimodozono M, Ogata A, et al.: Addition of intensive repetition of facilitation exercise to multidisciplinary rehabilitation promotes motor functional recovery of the hemiplegic lower limb. J Rehabil Med, 2004, 36: 159-164. [Medline] [CrossRef]

16) Kawahira K: Exercise therapy for motor recovery from hemiplegia: Theory and technique of repetitive facilitative exercise. Text with DVD-Video, 2nd ed. Tokyo: Igaku-shoin, 2010. (in Japanese).

17) Chino N, Melvin JL: Functional Evaluation of Stroke Patients. Springer-Verlag, Japan, 1995, pp 19-31.

18) Yamanaka H, Kawahira K, Arima M, et al.: Evaluation of skilled arm movements in patients with stroke using a computerized motor-skill analyser for the arm. Int J Rehabil Res, 2005, 28: 277-283. [Medline] [CrossRef]

19) Bohannon RW, Smith MB: Interrater reliability of a modified Ashworth scale of muscle spasticity. Phys Ther, 1987, 67: 206-207. [Medline]

20) Waters RL, Adkins RH, Yakura JS, et al.: Motor and sensory recovery following incomplete tetraplegia. Arch Phys Med Rehabil, 1994, 75: 306-311. [Medline] [CrossRef]

21) Ditunno JF Jr, Cohen ME, Hauck WW, et al.: Recovery of upper-extremity strength in complete and incomplete tetraplegia: a multicenter study. Arch Phys Med Rehabil, 2000, 81: 389-393. [Medline] [CrossRef]

22) Antonov I, Antonova I, Kandel ER, et al.: Activity-dependent presynaptic facilitation and hebbian LTP are both required and interact during classical conditioning in Aplysia. Neuron, 2003, 37: 135-147. [Medline] [CrossRef] 